Reprod. Nutr. Dévelop., 1983, 23 (3), 537-552.

\title{
Caractéristiques des réactions de précipitation entre les produits de sécrétion de l'oviducte du Pleurodèle : identification d'une lectine
}

\author{
P. JĖGO, A. CHESNEL, H. LERIVRAY, H. LE TALLEC
}

Laboratoire de Biologie de /a Reproduction, (C.N.R.S. LA 256),

Université de Rennes I, 35042 Rennes Cedex, France.

Summary. The properties of precipitation reactions between secretion products in the oviduct of Pleurodeles : identification of a lectin.

The properties of two precipitation reactions occurring between secretory products from the oviduct of Pleurodeles walt have been studied. It has been demonstrated that a lectin is involved in one of the reactions. This lectin precipitated glycogen and starch and required calcium; the most potent saccharide inhibitors were 2-amino-2-deoxy-D-glucose and D-glucose, respectively.

The other reaction was related to glycoproteins (probably sulfated glycoproteins) that contained sulphur. The properties of this reaction were not the same as purely ionic interactions; basic protein-acidic polysaccharide interactions have been compared. A lectin was probably implicated but this could not be demonstrated because no saccharide inhibitor was found.

There are several similitudes between this reaction and the lectin-galactoside reaction which occurs in the reaction between cortical granule content and egg jellies in anurans.

\section{Introduction}

Les gangues ovulaires d'Amphibiens ont retenu l'attention des auteurs depuis fort longtemps. Au début du siècle, les travaux de Bataillon (1919) qui furent confirmés plus tard par d'autres auteurs (voir revue de Metz, 1967), mettaient en évidence un rôle physiologique de ces gangues dans la fécondation. L'hypothèse avancée par Soupart et Noyes (1964) pour les Mammifères et reprise par Humphries (1966) pour les Amphibiens, d'un rôle possible des glucides (acides sialiques en particulier) dans les phases préliminaires à la fécondation, risquait de trouver un champ expérimental favorable chez les Amphibiens puisque de nombreux travaux montrent la richesse en glucides de ces produits d'excrétion de l'oviducte (Folkes et al., 1950 ; Minganti et d'Anna, 1957, 1958 ; Bolognani et al., 1966 ; Humphries, 1966 ; Lee, 1967 ; Mendez et al., 1968 ; Katagiri, 1973 ; Boisseau et al., 1974 ; Jégo, 1974 ; Yurewicz et al., 1975).

Wyrick et al. (1974), ont montré que le contenu des granules corticaux des ovocytes de Xenopus laevis (Amphibien, Anoure) réagissait avec certains consti- 
tuants de la gangue interne, pour former un précipité visible sous forme d'arc en gel d'agarose. Nous avons nous même montré que certains produits d'excrétion de l'oviducte de Pleurodeles walt/ (Amphibien, Urodèle) réagissent entre eux pour former des précipitations " de type antigène-anticorps " (Jégo et al., 1976).

Dans un précédent article, nous montrons qu'il existe en réalité dans l'oviducte antérieur, puis dans la gangue ovulaire interne du Pleurodèle au moins deux catégories de molécules capables de produire des réactions de précipitation (Jégo et al., 1983). Ces molécules ont été appelées $L_{1}$ et $L_{2}$; elles réagissent avec des produits sécrétés par l'oviducte moyen (respectivement $I_{1}$ et $I_{2}$ ). Dans le présent travail, nous donnons les principales caractéristiques des réactions entre ces molécules que nous avons appelées réactions $L_{1}-I_{1}$ et réactions $L_{2}-I_{2}$.

\section{Matériel et méthodes}

1) Extraits d'oviducte. - Les animaux sont des femelles adultes de Pleurodeles walt/ Michah, élevées au laboratoire. Les segments antérieurs et les segments moyens de l'oviducte sont prélevés puis homogénéisés dans un tampon qui est, sauf cas particuliers (précisés dans le paragraphe "Résultats »), du tampon tris- $\mathrm{HCl} 0,1 \mathrm{M}, 10 \mathrm{mM}$ en $\mathrm{Ca}^{+}{ }^{+}$, à $\mathrm{pH} 7$ ou à $\mathrm{pH} 8,2$ (les deux valeurs du $\mathrm{pH}$ ont été systématiquement essayées) à raison de deux segments pour $4 \mathrm{ml}$ de tampon. Ces homogénats sont centrifugés à $20000 \mathrm{~g}$ pendant $1 \mathrm{~h}$; les surnageants obtenus constituent les extraits d'oviducte et seront dénommés respectivement OA et OM ou $A$ et $M$.

2) Dosages néphélémétriques. - Nous avons appelé réaction globale OA$O M$, la réaction obtenue en mélangeant les extraits $O A$ et les extraits $O M$ dans un tube à essai. Après incubation pendant $2 \mathrm{~h}$ à température ambiante (sauf exceptions précisées dans le paragraphe "Résultats "), l'intensité de la réaction est déterminée en mesurant la densité optique du mélange à $500 \mathrm{~nm}$.

Nous avons appelé réaction $L_{2}$-glycogène (ou $L_{2}$-amidon), la réaction obtenue en mélangeant l'extrait $O A$ avec une solution de glycogène à $10 \mathrm{mg} / \mathrm{ml}$ dans le tampon d'homogénéisation lou avec une solution d'amidon soluble obtenue en diluant 3 fois une solution d'amidon soluble à saturation).

3) Double diffusion sur ge/ d'agarose. - Les réactions de précipitation sont obtenues de manière classique (réactions de type Ouchterlony, 1949) sur de l'agarose à $1 \%$ dans le tampon d'homogénéisation après confrontation des extraits $O A$ et $O M$ et des solutions de glycogène ou d'amidon.

L'effet des différents facteurs a été analysé de deux manières : - soit par traitement des produits réactionnels par ces facteurs avant qu'ils ne soient déposés sur la lame d'agarose (dans la mesure du possible, les facteurs continuent à être appliqués pendant la diffusion sur agarose par traitement de celui-ci) ; soit par traitement des lames d'agarose sur lesquelles des arcs de précipitation

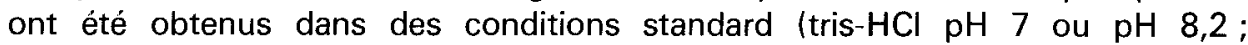
$\mathrm{Ca}^{++} 10 \mathrm{mM}$; $48 \mathrm{~h}$ d'incubation à $37^{\circ} \mathrm{C}$ en chambre humide ; lavage des lames 
dans le tampon pendant $48 \mathrm{~h}$ ). Dans le premier cas on étudiera l'apparition éventuelle des arcs de précipitation ; dans le second cas on étudiera leur disparition. Dans ces expériences de double diffusion, deux catégories de réactions nous ont servi de repères : d'une part des réactions de précipitation entre la concanavaline $A$ et le glycogène ; d'autre part des réactions de précipitation intervenant entre des protéines basiques (nous avons utilisé des histones et du sulfate de protamine) et des polysaccharides acides (acide alginique, acide polygalacturonique). Ces dernières réactions ne font intervenir que des interactions ioniques non spécifiques ; elles provoquent cependant des arcs de précipitation sur gel d'agarose.

4) Etude des effets inhibiteurs des saccharides. - Les effets inhibiteurs des saccharides sur les réactions de précipitation ont été étudiés qualitativement et semi-quantitativement sur des réactions obtenues sur gel d'agarose puis semiquantitativement et quantitativement sur des réactions obtenues en tubes à essai.

\section{5) Produits chimiques.}

a) Glucides. - Les saccharides suivants proviennent de chez Sigma :

D-arabinose, D-ribose, 2-désoxy-D-ribose, D-xylose.

L-fucose, L-rhamnose.

D-galactose, D-glucose, D-mannose, D-fructose.

2-amino-2-désoxy-D-galactose (D-galactosamine), 2-amino-2-désoxy-D-glucose (D-glucosamine), 2-amino-2-désoxy-D-mannose (D-mannosamine).

2-acétamido-2-désoxy-D galactose ( $\mathrm{N}$-acétyl-D-galactosamine) 2-acétamido-2désoxy-D-glucose ( $\mathrm{N}$-acétyl-D-glucosamine).

Acide D-galacturonique, acide D-glucuronique (lactone), acide D-galactonique (lactone), acide $\mathrm{D}$-gluconique (lactone), acide $\mathrm{N}$-acétyl-neuraminique.

D-cellobiose, $\alpha$-lactose, maltose, $\alpha$-D-mélibiose, saccharose, D-tréhalose.

D-mélézitose, D-raffinose.

Les polysaccharides suivants ont été utilisés : agarose, amidon soluble, amidon insoluble, glycogène (Merck) ; kappa-carragénine, lambda-carragénine, iotacarragénine, pectine, chondroïtine-sulfate (extrait de cartilages), acides alginique, hyaluronique (extrait de cordons ombilicaux) et polygalacturonique (Sigma) ; agar noble (Difco). L'agarose utilisé pour la confection des lames de double diffusion provient de chez IBF (indubiose = agarose A37).

b) Autres produits. - La pronase, la trypsine et l'inhibiteur de la trypsine lovomucoïde) proviennent de chez Boehringer-Mannheim. La concanavaline A provient de chez IBF. L'EDTA, I'EGTA, I'histone (extrait de thymus de veau) et le sulfate de protamine (extrait du hareng) proviennent de chez Sigma.

6) Expériences d'autoradiographie. - Dans ces expériences, les extraits OA et $\mathrm{OM}$ proviennent d'animaux qui ont reçu $16 \mathrm{~h}$ avant le sacrifice, $250 \mu \mathrm{Ci}$ de sulfate de sodium radioactif par voie cutanée $\left(\mathrm{Na}_{2}{ }^{35} \mathrm{SO}_{4},>100 \mathrm{~m} \mathrm{Ci} / \mathrm{mole}\right.$, Amersham). Après obtention des arcs sur les gels d'agarose, ceux-ci sont rincés 
longuement puis séchés avant d'être mis en contact, à l'obscurité, avec un film Kodirex (Kodak) pendant 15 jours. Les lames sont ensuite colorées au Noir Amide.

7) Dosage des protéines et des glucides; protéolyses. - Les protéines ont été dosées selon la méthode de Lowry (1951) et les glucides par la méthode à I'orcinol sulfurique (Tillmans et Philippi modifiée par Rimington, 1940). Les expériences de protéolyse ont été réalisées grâce à de la pronase ou de la trypsine (1 à $10 \mathrm{mg} / \mathrm{ml} ; 37^{\circ} \mathrm{C} ; 2$ à $6 \mathrm{~h}$ d'incubation); la trypsine est ensuite inactivée par addition d'un inhibiteur (ovomucoïde 10 à $50 \mathrm{mg} / \mathrm{ml}$ ) et la pronase par dénaturation thermique.

\section{Résultats.}

1) Effet du temps d'incubation (fig. 1). - La réaction globale OA-OM est assez rapide ; l'intensité maximale est obtenue après 20 min environ. La réaction $L_{2}$-glycogène ou $L_{2}$-amidon est plus lente à se développer puisqu'il faut environ $1 \mathrm{~h}$ pour qu'elle soit maximale. Une fois formés, les produits des réactions sont stables pendant $12 \mathrm{~h}$ au moins à $4{ }^{\circ} \mathrm{C}$.

Sur gel d'agarose, les arcs $L_{1}-L_{1}$ apparaissent après 10 à $12 \mathrm{~h}$ d'incubation, mais ils ne sont très intenses qu'après 24 à $36 \mathrm{~h}$ d'incubation. Les arcs $\mathrm{L}_{2}-\mathrm{I}_{2}$ apparaissent le plus souvent en même temps que les précédents alors que les arcs $L_{2}$-glycogène ou $L_{2}$-amidon sont déjà visibles après 6 à $8 h$ d'incubation.

2) Effet des quantités respectives de produits réactionnels. - On obtient une intensité maximale pour la réaction globale OA-OM lorsqu'on mélange volume à volume des surnageants $\mathrm{OA}$ et $\mathrm{OM}$ renfermant les mêmes concentrations en protéines.

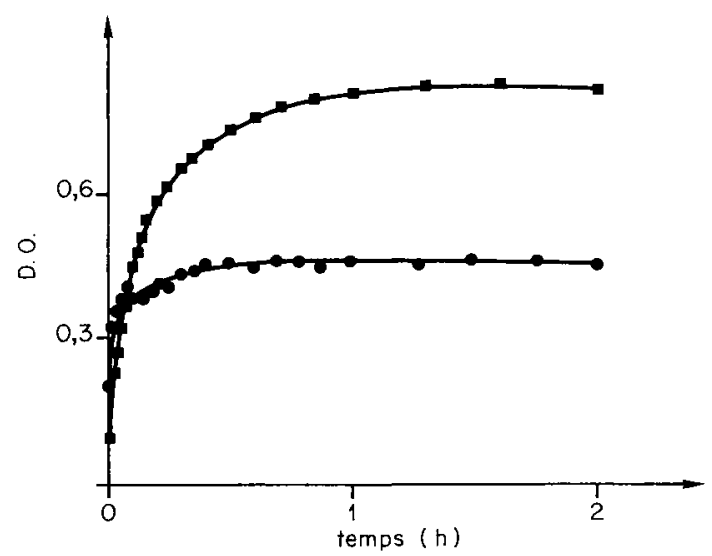

FIG. 1. - Effet du temps d'incubation sur les réactions de précipitation. (Dosages néphélémétriques $-\mathrm{pH} 8,2-25^{\circ} \mathrm{C}$. 
Les réactions $L_{2}$-amidon et $L_{2}$-glycogène s'effectuent de façon optimale lorsque le rapport de la concentration en protéines de l'extrait d'oviducte antérieur, à la concentration en glucose de la solution d'amidon ou de glycogène, est voisin de 2. On obtient alors une fine suspension blanchâtre qui sédimente lentement. Si ce rapport est légèrement différent de 2 (de 1,5 à 2,5 environ), on obtient une suspension de grosses particules qui sédimentent rapidement ; $\mathrm{s}^{\prime} i l$ est nettement différent de 2, il n'y a aucun précipité.

Sur gel d'agarose, lorsqu'on confronte les extraits dans les conditions de concentrations optimales indiquées ci-dessus, les arcs de précipitation se forment à égale distance des deux puits de dépôt pour $L_{1}-I_{1}, L_{2}$-amidon ou $L_{2}$-glycogène ; les arcs $L_{2}-I_{2}$ se forment par contre toujours très près du puits de dépôt des extraits $\mathrm{OM}$.

3) Effet des ions. - Nous n'avons trouvé aucun ion qui soit capable de favoriser la réaction $L_{1}-I_{1}$; même à fortes concentrations, I'EDTA et I'EGTA ne modifient pas les arcs de précipitation $L_{1}-l_{1}$ sur gel d'agarose.

Les ions $\mathrm{Ca}^{++}$sont indispensables aux réactions $L_{2}-L_{2}, L_{2}$-glycogène et $\mathrm{L}_{2}$-amidon (fig. 2) ; la concentration optimale est voisine de $10 \mathrm{mM}$. Le manganèse et le magnésium ne remplacent pas le calcium. On peut sans difficulté faire apparaître puis disparaître les précipités $L_{2}$-amidon ou $L_{2}$-glycogène par actions successives de solutions d'EGTA ou d'EDTA et de calcium ; la réversibilité de la réaction est pratiquement instantanée (même sur agarose).

A titre de comparaison, nous avons essayé l'effet de différents ions sur des réactions de précipitation obtenues sur lame d'agarose après confrontation de protéines basiques (protamines ou histones) avec des polysaccharides acides (acides polygalacturonique et alginique en particulier). Ces réactions donnent le plus souvent des arcs de précipitation qui disparaissent progressivement en pré-

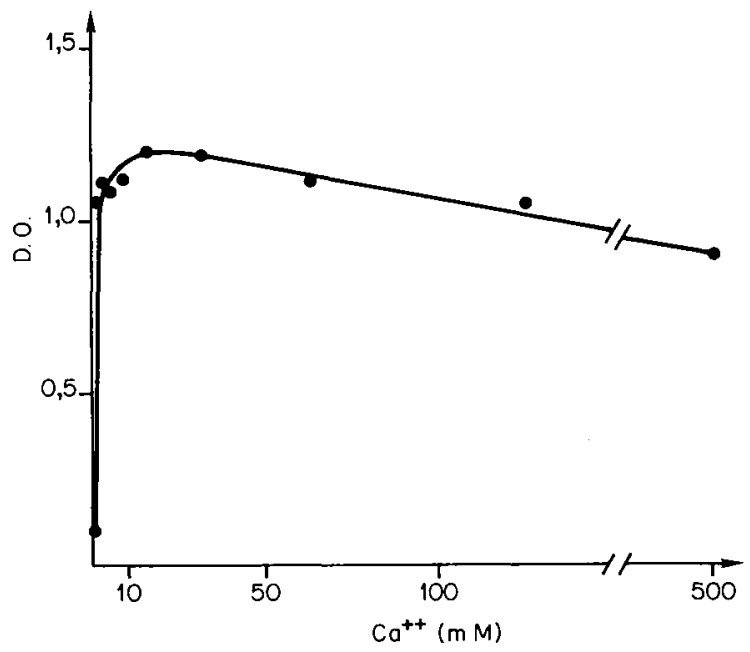

FIG. 2. - Effet du calcium sur la réaction $L_{2}$-amidon. (Dosages néphélémétriques $-\mathrm{pH} 8,2-$ $-25{ }^{\circ} \mathrm{C}-2 \mathrm{~h}$ d'incubation). 
sence de concentrations croissantes de chlorure de sodium, de chlorure de calcium ou de sulfate de sodium ; l'inhibition est totale dans tous les cas pour des concentrations de 100 à $200 \mathrm{mM}$. Placés dans les mêmes conditions, les arcs $L_{1}-I_{1}, L_{2}-I_{2}, L_{2}$-amidon ou $L_{2}$-glycogène, restent intacts.

4) Effet $d u p H$ (fig. 3 et 4). - La réaction globale OA-OM, bien qu'assez peu sensible au $\mathrm{pH}$, présente un maximum aux alentours de $\mathrm{pH}$ 7. Aux pH extrê-

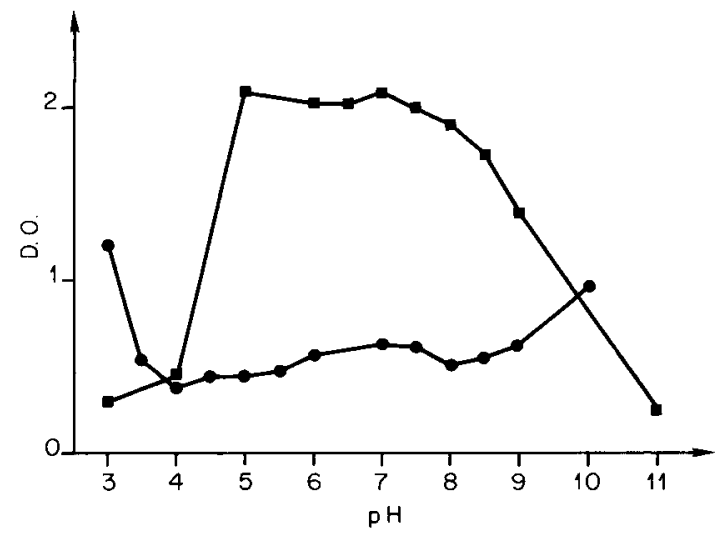

FIG. 3. - Effet du $\rho H$ sur les réactions de précipitation. (Dosages néphélémétriques $-25^{\circ} \mathrm{C}-2 \mathrm{~h}$ d'incubation $-\mathrm{Ca}^{++} 1,25 \mathrm{mM}$ ).

Les différents $\mathrm{pH}$ ont été obtenus avec des tampons acétate de sodium - acide acétique de $\mathrm{pH}$ 3 à $\mathrm{pH}$ 6,5 et des tampons tris - acide chlorhydrique de $\mathrm{pH} 7$ à $\mathrm{pH} 11$.

Réaction globale OA-OM ; Réaction $\mathrm{L}_{2}$-amidon.

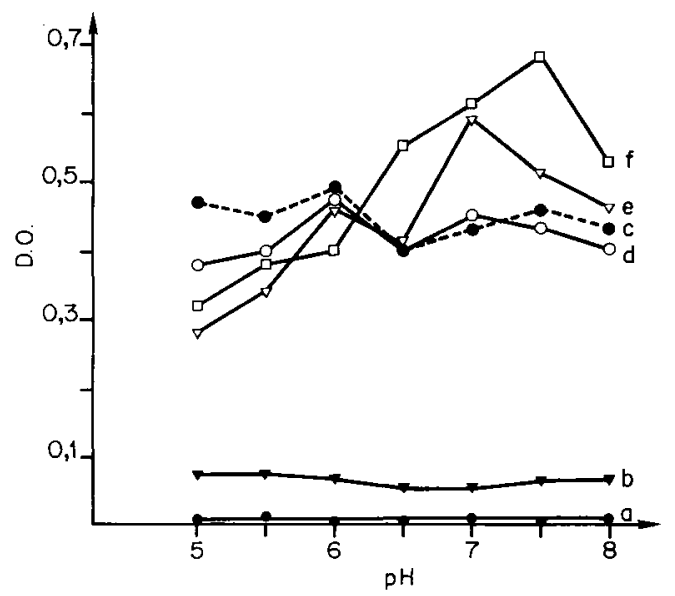

FIG. 4. - Effets combinés du $\rho H$ et $d u$ calcium sur la réaction $L_{2}$-amidon. (Dosages néphèlemétriques $-25{ }^{\circ} \mathrm{C}-2 \mathrm{~h}$ d'incubation).

a : amidon seul ; b : extrait d'oviducte antérieur seul ; c à f : extrait d'oviducte antérieur + amidon à des concentrations croissantes en $\mathrm{Ca}^{+}+(1,25 \mathrm{mM}$ en $\mathrm{c} ; 2,05 \mathrm{mM}$ en $\mathrm{d} ; 2,85 \mathrm{mM}$ en e ; $3,75 \mathrm{mM}$ en $\mathrm{fl}$. 
mes, on observe une précipitation non spécifique de certains produits de sécrétion, de l'oviducte moyen notamment.

Sur lame d'agarose, les arcs de précipitation $L_{1}-I_{1}$ et $L_{2}-I_{2}$ apparaissent dès que le $\mathrm{pH}$ est supérieur à 4 et ne disparaissent que pour des $\mathrm{pH}$ supérieurs à 9 . Entre ces deux valeurs, on ne note aucune variation décelable dans la position et l'intensité des arcs de précipitation. Cependant, lorsque le pH est compris entre 5,5 et 7,5 , des halos très marqués apparaissent autour des puits de dépôt des extraits $\mathrm{OA}$; ces halos n'existent plus à pH 4,5 ou à $\mathrm{pH} 8$. Ces halos gênent parfois l'observation des arcs de précipitation, c'est pourquoi systématiquement, nos expériences ont été réalisées à $\mathrm{pH} 7$ et à $\mathrm{pH} \mathrm{8,2}$.

$\mathrm{La}$ réaction $\mathrm{L}_{2}$-amidon se déroule convenablement pour des $\mathrm{pH}$ allant de 5 à 8 . II existe cependant deux légers maxima à pH 5-6 d'une part et à $\mathrm{pH} 7$ d'autre part. Si l'on ajoute du calcium au milieu réactionnel, le premier pic (pH 56) s'estompe nettement, tandis que le second $(\mathrm{pH} 7)$ augmente très sensiblement (fig. 4). A ces variations quantitatives de l'intensité de la réaction obtenue en tube à essai sous les effets combinés du $\mathrm{pH}$ et de la concentration en $\mathrm{Ca}^{++}$, nous $\mathrm{n}^{\prime}$ avons jamais pu associer de modifications qualitatives des arcs obtenus sur gel d'agarose dans les mêmes conditions.

5) Effet de la température (fig. 5 et 6). - La réaction globale OA-OM dépend peu de la température. $L^{\prime}$ intensité de la réaction $L_{2}$-amidon est par contre multipliée par 5 entre $0{ }^{\circ} \mathrm{C}$ et $40^{\circ} \mathrm{C}$. Le maximum d'intensité est obtenu à $60{ }^{\circ} \mathrm{C}$ environ. Cependant, la $\mathrm{D}$-glucosamine qui inhibe la réaction $\mathrm{L}_{2}$-amidon (voir paragraphe suivant), a une efficacité croissante de $0^{\circ} \mathrm{C}$ à $40{ }^{\circ} \mathrm{C}$; l'inhibition qui est maximale aux alentours de $40^{\circ} \mathrm{C}-50^{\circ} \mathrm{C}$ diminue très nettement pour $60^{\circ} \mathrm{C}$ et $70^{\circ} \mathrm{C}$ (fig. 6). II est donc fort probable qu'au-dessus de $50^{\circ} \mathrm{C}$ apparaissent des précipitations non spécifiques qui perturbent les dosages.

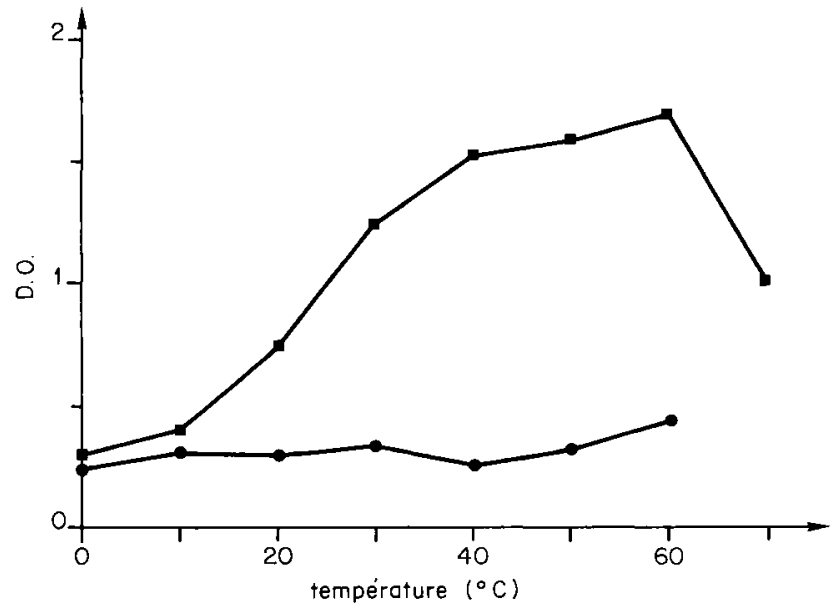

FIG. 5. - Effet de la température sur les réactions de précipitation. (Dosages néphélémétriques $-\mathrm{pH} 7-2 \mathrm{~h}$ d'incubation $-\mathrm{Ca}^{++} 1,25 \mathrm{mM}$ ).

Réaction globale OA-OM; Réaction $\mathrm{L}_{2}$-amidon. 


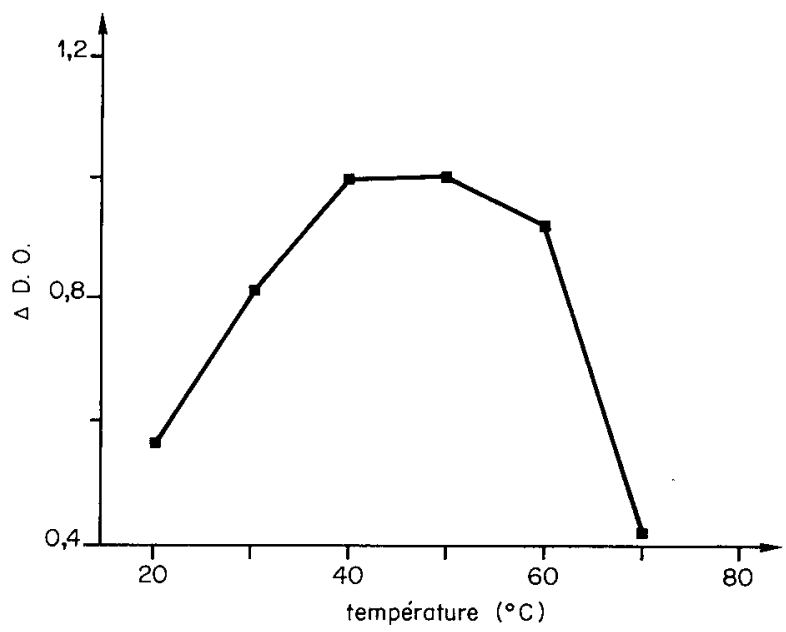

FIG. 6. - Effets combinés de la température et de la D-glucosamine sur la réaction $L_{2}$-amidon. (Dosages néphélémétriques $-\mathrm{pH} 7-2 \mathrm{~h}$ d'incubation $-\mathrm{Ca}^{+}+1,25 \mathrm{mM}$ ).

$\triangle \mathrm{DO}$ : Diminution de l'intensité de la réaction obtenue en ajoutant de la D-glucosamine (10 $\mathrm{mM})$ au milieu.

Aucune des deux réactions de précipitation ne se produit sur gel d'agarose si l'on porte au préalable à $100^{\circ} \mathrm{C}$ pendant 1 à 2 min les extraits OA. Les composés $l_{1}$ et $I_{2}$ supportent, par contre, très bien de telles dénaturations thermiques même si elles durent 10 à $20 \mathrm{~min}$.

Les protéines basiques et les polysaccharides acides supportent très bien un traitement à $100^{\circ} \mathrm{C}$ pendant 10 à 15 min sans modification des arcs de précipitation.

\section{6) Action des glucides}

a) Effets inhibiteurs des mono-, di- ou tri-saccharides

Parmi les saccharides que nous avons utilisés (voir "Matériel et méthodes $\%$ ), aucun n'exerce un effet inhibiteur net et constant sur la réaction $\mathrm{L}_{1} \mathrm{I}_{1}$.

Les réactions $L_{2}-t_{2}, L_{2}$-glycogène et $L_{2}$-amidon, sont par contre, très inhibées par les saccharides suivants (entre parenthèses les concentrations qui produisent $50 \%$ d'inhibition): D-glucosamine (2-amino-2-désoxy-D-glucose) $(6 \mathrm{mM})$, Dglucose $(13 \mathrm{mM})$; ces réactions sont moyennement inhibées par le saccharose $(44 \mathrm{mM})$ et le maltose $(40 \mathrm{mM})$; elles sont un peu inhibées par le D-xylose, le Dfructose, le $\alpha$-D-mélibiose et le D-raffinose $(>100 \mathrm{mM})$. En revanche, elles sont complètement indifférentes aux autres saccharides utilisés.

b) Précipitation avec les polysaccharides (PI. I)

Parmi les polysaccharides que nous avons utilisés, aucun ne réagit avec les produits $L_{1}$ de manière à former des arcs en communauté avec les arcs $L_{1}-I_{1}$. 

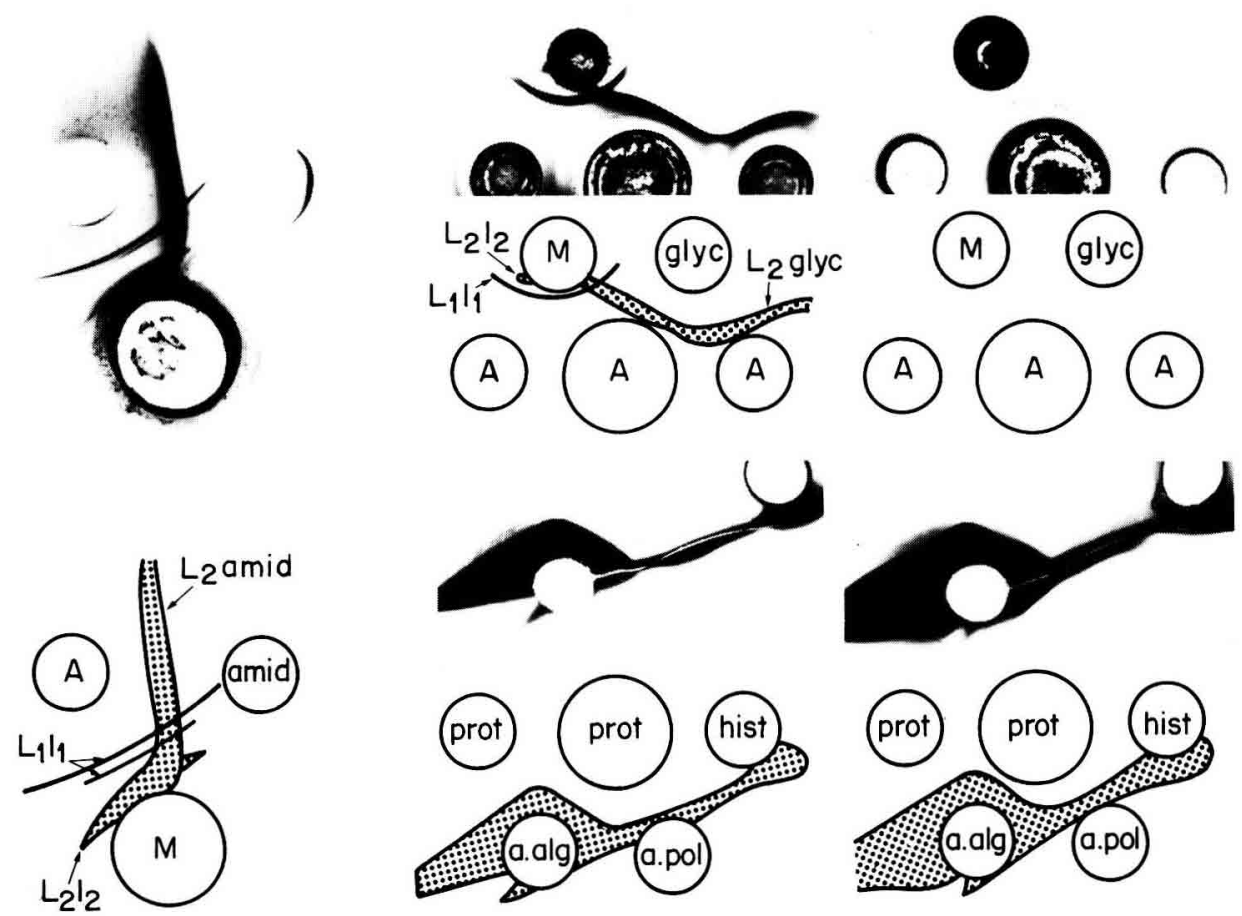

PLANCHE ।

Réactions de précipitation avec les polysaccharides.

(A : extrait d'oviducte antérieur, $M$ : extrait d'oviducte moyen)

à gauche : Photographie montrant la communauté entre les arcs $L_{2}{ }_{2} I_{2}$ et les arcs $L_{2}$-amidon lamid = amidon).

au centre et a droite : Effet de la dénaturation thermique sur les réactions $L_{1} L_{1}, L_{2}-L_{2}$ et $L_{2}$-glycogène (en haut à gauche : témoin ; en haut à droite : les extraits ont été portés à $100^{\circ} \mathrm{C}$ pendant 10 $\min$ )

et sur les réactions protéines basiques - polysaccharides acides (en bas à gauche : témoin ; en bas à droite : $100{ }^{\circ} \mathrm{C}$ pendant $10 \mathrm{~min}$ ).

glyc : glycogène ; prot : sulfate de protamine; his : histones; a. alg : acide alginique ; a. pol : acide polygalacturonique.

Le glycogène et l'amidon forment des arcs de précipitation très nets avec les extraits OA ; ces arcs sont en communauté avec les arcs majeurs $L_{2} \mathrm{I}_{2}$. L'agarose, confronté aux extraits OA forme très souvent des arcs très faibles qui semblent en communauté (au moins partielle) avec les arcs $\mathrm{L}_{2}-\mathrm{I}_{2}$. Les polysaccharides légèrement acides ou très acides (chondroïtine sulfate, carragénine, pectine, acides hyaluronique, polygalacturonique, alginique et la gomme arabique) forment avec les extraits $O A$, des arcs de précipitation qui semblent parfois en communauté partielle avec les arcs $L_{2}-I_{2}$, mais présentent les caractéristiques des arcs résultant de réactions de précipitation dues à des interactions ioniques non spécifiques (type protéine basique-polysaccharide acide). Ces arcs se produisent d'ailleurs parfois contre les extraits OM. 


\section{7) Intervention de composés soufrés}

\section{a) Effet des mercaptans}

Le mercaptoéthanol et le dithiothréitol (à la concentration de $100 \mathrm{mM}$ ) empêchent la formation des arcs $L_{1}{ }^{-} I_{1}, L_{2}{ }^{-} I_{2}, L_{2}$-glycogène et $L_{2}$-amidon, lorsqu'ils sont ajoutés au début des réactions. Ils sont aussi capables de faire disparaître des arcs $L_{2}-I_{2}, L_{2}$-glycogène ou $L_{2}$-amidon préalablement formés sur gel d'agarose. En revanche, ils ne touchent pas du tout les arcs $L_{1}-I_{1}$ préalablement formés (même après un temps de contact de plusieurs jours).

\section{b) Marquage au ${ }^{35} \mathrm{~S}$ (pl. II)}

Les arcs $L_{1}-I_{1}$ sont radioactifs alors que les arcs $L_{2}{ }^{-} I_{2}$ ne le sont pas. On note une très forte radioactivité autour des puits de dépôt des extraits $O A$, mais pas autour de ceux des extraits OM.

Confrontés à un sérum de lapin immunisé contre les produits excrétés par OA (gangue ovulaire interne), les extraits $O A$ et $O M$ ne forment aucun arc d'immunoprécipitation radioactif. Confrontés à un sérum dirigé contre les produits excrétés par OM (gangue moyenne), les extraits OA ne forment aucun arc d'immunoprécipitation radioactif ; les extraits OM forment par contre deux arcs, I'un qui est étranger aux réactions $L_{1}-I_{1}$ ou $L_{2}-I_{2}$ et $I^{\prime}$ autre qui résulte de la reconnaissance, par certains anticorps, des produits $I_{1}$. Les arcs $L_{1} I_{1}$ radioactifs sont très nettement déplacés en présence de ce deuxième sérum.

II apparaît ainsi que deux antigènes de l'oviducte moyen, dont $l_{1}$, ont incorporé du soufre alors qu'aucun antigène de l'oviducte antérieur (ni $L_{1}, n$ i $L_{2}$ en particulier) n'en a incorporé. Ce résultat est d'autant plus significatif que les comptages de radioactivité que nous avons effectués dans les différentes régions de l'oviducte, indiquent que c'est l'oviducte antérieur qui incorpore le plus de soufre radioactif : ramenés à la même quantité de protéines, les proportions de soufre total incorporé sont approximativement de 6, 2, 1 respectivement pour les régions antérieure, moyenne et postérieure. [Ceci étant conforme à ce qui avait été observé précédemment par Boisseau (1973), sur l'incorporation du ${ }^{35}$ S dans les différentes régions de l'oviducte et à la distribution du sulfate dans les différentes régions de cet organe (Jégo, 1976)].

8) Nature glycoprotéique des principales molécules intervenant dans les précipitations. - Les arcs de précipitation sont tous colorés, à la fois par les colorants des protéines (noir-amide $10 \mathrm{~B}$ ) et des glycoprotéines (acide périodiquecolorant de Nadi) (Uriel, 1971).

Si l'on traite les extraits OA ou OM par les enzymes protéolytiques (pronase à $1 \mathrm{mg} / \mathrm{ml}$ ou trypsine à $10 \mathrm{mg} / \mathrm{ml}$ ), avant de les déposer dans les puits de gel d'agarose, il n'apparaît plus aucun arc. La pronase est beaucoup plus efficace que la trypsine, même à concentration dix fois moins forte. Si l'on traite par la pronase $(1 \mathrm{mg} / \mathrm{ml})$ des gels d'agarose sur lesquels on $a$, au préalable, fait se développer les différents arcs, on observe la disparition en $12 \mathrm{~h}$ environ de tous les arcs $L_{1}-I_{1}$ alors que les arcs $L_{2}-I_{2}, L_{2}$-amidon ou $L_{2}$-glycogène, persistent sans modification visible après 4 jours de traitement. 

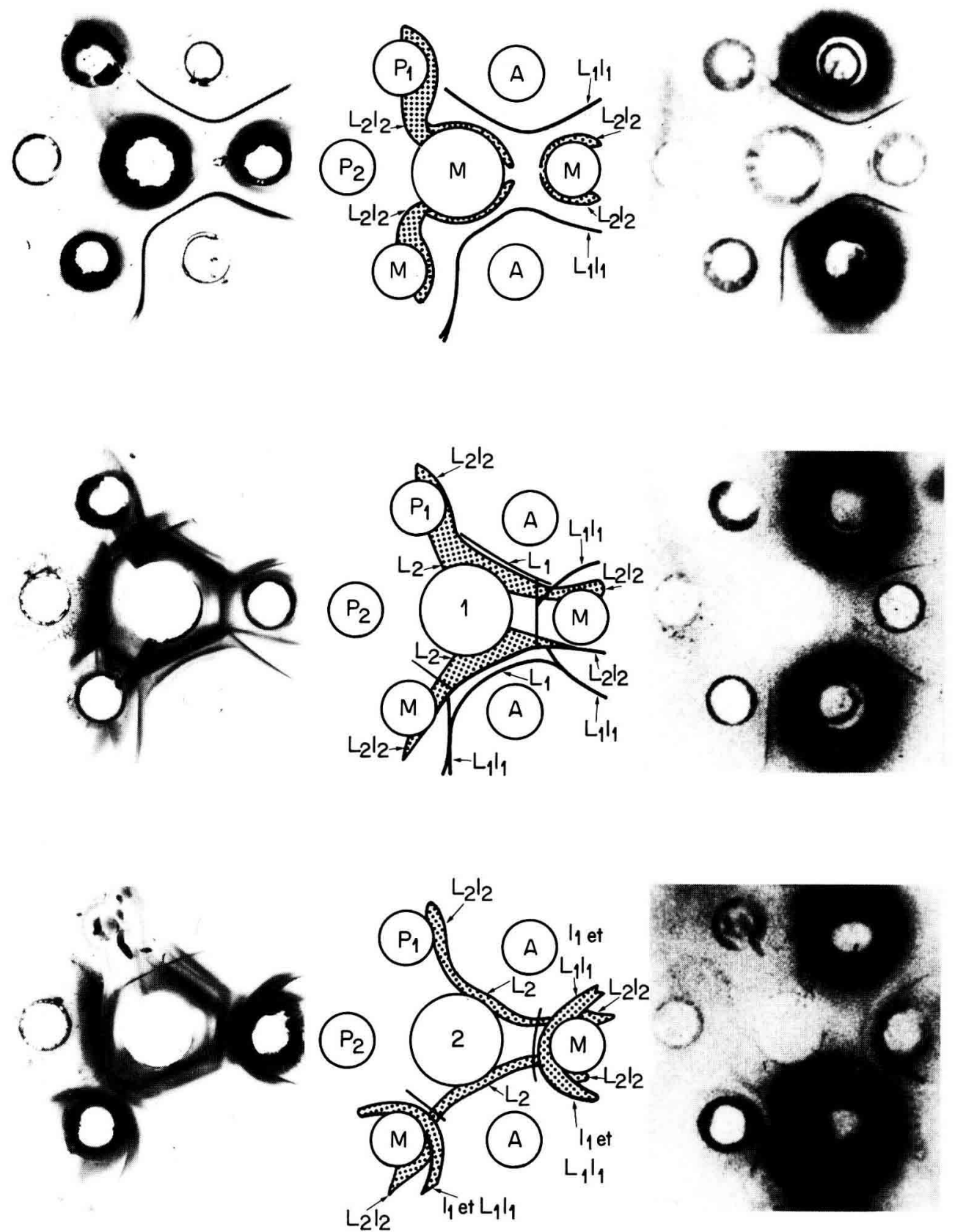

PLANCHE II

Incorporation de soufre $\left(^{35} \mathrm{~S}\right.$ ) dans les produits de sécrétion intervenant dans les réactions de précipitation.

Les animaux ont reçu une injection de $\mathrm{Na}_{2} \mathrm{SO}_{4}$ radioactif $16 \mathrm{~h}$ avant le sacrifice ; les différents segments de l'oviducte sont alors prélevés et les extraits sont préparés.

$(A, M$ : segments antérieur et moyen ; $P 1, P 2$ régions antérieures et postérieures du segment postérieur).

à droite : Autoradiographies des gels sur lesquels les extraits sont confrontés entre eux (en haut), au sérum 1 dirigé contre la gangue ovulaire interne (au milieu) ou au sérum 2 dirigé contre la gangue ovulaire moyenne (en bas).

à gauche: Photographies des mêmes ge/s colorés au noir-amide.

au milieu: Schémas sur lesquels ne sont portés que les arcs reconnus de façon certaine. $L_{1} l_{1}$ et $L_{2} l_{2}$ désignent les arcs de précipitation. $L_{1}, L_{2}, l_{1}$ désignent les arcs d'immunoprécipitation. 
9) Réactions de précipitation avec la gangue ovulaire interne (fig. 7). - Les expériences ont été réalisées avec de la gangue ovulaire interne entourant des ovocytes déposés dans l'eau depuis $15 \mathrm{~h}$ environ ; celle-ci a été minutieusement séparée des autres gangues, lyophilisée puis mis en solution dans du tampon tris- $\mathrm{HCl} 0,1 \mathrm{M}$ à pH 7 .

(A)

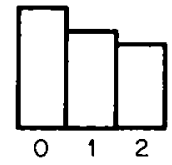

(B)

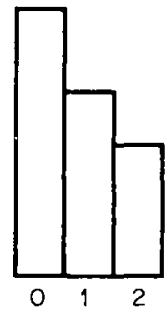

glucosomine
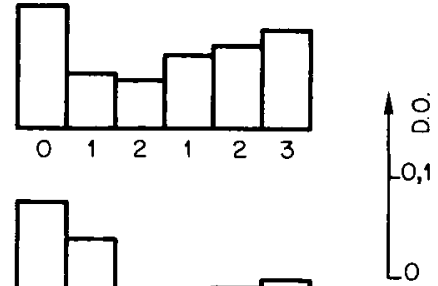

FIG. 7. - Réaction $L_{2}$-amidon avec la gangue ovulaire interne. (Dosages néphélémétriques - $\mathrm{pH} 7$

$-2 \mathrm{~h} \mathrm{~d}^{\prime}$ incubation).

De la gangue ovulaire interne seule ( $A$ ) ou mélangée avec de l'amidon (B) est mise en présence de D-glucosamine à des concentrations croissantes $(0=0 \mathrm{mM} ; 1=4 \mathrm{mM} ; 2=8 \mathrm{mM})$, d'EGTA $0=0 \mathrm{mM} ; 1=10 \mathrm{mM} ; 2=20 \mathrm{mM})$ ou d'EGTA et de calcium $(1,2$ et $3:$ EGTA = $\left.20 \mathrm{mM} ; 1: \mathrm{Ca}^{++}=10 \mathrm{mM} ; 2: \mathrm{Ca}^{++}=20 \mathrm{mM} ; 3: \mathrm{Ca}^{++}=50 \mathrm{mM}\right)$.

La gangue ovulaire interne seule présente une certaine densité optique qui peut être très nettement augmentée par l'addition d'une solution d'amidon soluble. En présence, comme en absence d'amidon, la densité optique à $500 \mathrm{~nm}$ peut être significativement diminuée par la D-glucosamine et I'EGTA ; dans ce dernier cas, la réaction est réversible sous l'effet du calcium. Ces résultats montrent d'une part que les molécules $L_{2}$ ont conservé leurs propriétés de précipitation $15 \mathrm{~h}$ après la ponte des ovocytes dans l'eau; les molécules $L_{1}$ ne peuvent plus être mises en évidence aussi tardivement. Ils montrent d'autre part, que des réactions de type $L_{2}-I_{2}$ interviennent spontanément au sein de la gangue interne ; ces réactions ne neutralisent qu'une faible partie des molécules $L_{2}$ puisqu'il subsiste une précipitation importante en présence d'amidon.

\section{Discussion.}

Les différentes propriétés de la molécule $L_{2}$, mises en évidence dans ce travail, permettent de considérer qu'il s'agit d'une lectine. Si l'on tient compte du fait qu'elle reconnaît le $D$-glucose, cette lectine $L_{2}$ se rapproche des lectines extraites de Canavalia ensiformis (concanavaline A), Lens culinaris, Pisum sativum et Vicia faba ; elle s'en différencie cependant très nettement en ne reconnaissant 
pas le D-mannose. Comme les deux dernières, elle reconnaît le 2-amino-2désoxy-D-glucose et se distingue donc de la concanavaline $A$ et de la lectine extraite de Lens culinaris qui ne reconnaissent la D-glucosamine que lorsqu'elle est $\mathrm{N}$-acétylée (Goldstein et Hayes, 1978). En comparant la structure des pentoses et des hexoses qui exercent une inhibition sur la réaction $L_{2}$-glycogène, il apparaît que la nature et la position des atomes portés par les carbones 2, 3 et 4 jouent un rôle important dans la reconnaissance. Le fait que certains disaccharides comme le maltose, le saccharose et le mélibiose ou trisaccharides comme le raffinose aient une activité inhibitrice sur la réaction $L_{2}$-glycogène alors que d'autres comme le cellobiose, le lactose, le tréhalose et le mélézitose qui renferment pourtant au moins une molécule de D-glucopyranose sont inefficaces, suggère l'existence de plusieurs sites de reconnaissance contigus dans la molécule $\mathrm{L}_{2}$ comme cela a été montré pour beaucoup de lectines connues (Goldstein et Hayes, 1978 ; Wright, 1980).

Les réactions $L_{1}-I_{1}$ font intervenir des réactifs en proportions bien déterminées puisqu'elles se concrétisent sous forme d'arcs de précipitation en gel d'agarose ; ceci nous les avait fait nommer réactions de type " antigène-anticorps " (Jégo et al., 1976). Les réactions de précipitation $L_{1}-_{1} n^{\prime}$ ont pas les caractéristiques des précipitations qui résultent de simples interactions ioniques ; en outre, nous avons vu précédemment que les sites moléculaires de $l_{1}$ impliqués dans ces réactions sont des sites antigéniques communs à tous les Urodèles que nous avons étudiés (Jégo et al., 1983). On est donc amené à retenir l'hypothèse de reconnaissances moléculaires précises entre les produits $L_{1}$ et $I_{1}$. Parmi les composés localisés dans un même organe et qui sont susceptibles de réaliser de telles reconnaissances, ce sont les lectines qui sont les mieux connues. Aucun des saccharides étudiés n'exerce d'action inhibitrice sur la réaction $L_{1}-I_{1}$, mais nous n'avons pas essayé tous les saccharides qui existent dans la nature ; il existe d'autre part certaines lectines qui ne peuvent être inhibées que par des oligosaccharides compliqués (Phaseolus vulgaris) et d'autres dont on ne connaît pas d'inhibiteur saccharidique (Vicia graminea) (Goldstein et hayes, 1978).

Dans un autre travail (Jégo et al., 1983) nous comparons les réactions $L_{1}-I_{1}$, obtenues chez les Urodèles, aux réactions qui apparaissent entre le contenu des granules corticaux et la gangue interne chez les Anoures (Wyrick et al., 1974), dans la mesure où toutes les deux pourraient être responsables du blocage des spermatozoïdes. Les caractéristiques de ces deux catégories de réactions présentent également quelques similitudes : les composés $I_{1}$ renferment beaucoup de soufre, tout comme les glycoprotéines de la gangue interne qui réagissent avec le contenu des granules corticaux chez le Xénope (Birr et Hedrick, 1979). L'enveloppe de fécondation constituée par ce type de réactions chez le Xénope, présente la propriété de devenir progressivement insolubilisable par les mercaptans (Wolf, 1974), tout comme les arcs $L_{1}-l_{1}$ que nous avons obtenus. Nous pensons que les réactions $L_{1}-l_{1}$ résultent de la reconnaissance par une lectine $L_{1}$, de sites saccharidiques sulfatés portés par des glycoprotéines $\left(I_{1}\right)$. La nature des saccharides de ces sites, reste à déterminer.

Si les effets de la température et du $\mathrm{pH}$ sont le plus souvent assez complexes sur les réactions $L_{1}^{-I_{1}}$ et surtout $L_{2}{ }^{-} I_{2}$ (voir en particulier les effets combi- 
nés du $\mathrm{pH}$ et du calcium), cela provient probablement du fait que les molécules $L_{1}$ et $L_{2}$ se trouvent en majorité sous une forme polymérisée. Par chromatographie sur séphadex $\mathrm{G}_{100}$ à différents $\mathrm{pH}$, nous avons pu observer que ces molécules sont en très grande majorité éluées en même temps que le bleu dextran si on ne leur fait subir aucun traitement dénaturant (Jégo, 1978). Cette polymérisation spontanée expliquerait l'existence des granules que l'on peut observer dans la gangue interne juste après la ponte des œufs (Boisseau et al., 1974). On peut envisager plusieurs facteurs de polymérisation ; à côté du calcium, des reconnaissances de type lectines-saccharides (n'épuisant pas l'aptitude à réagir des molécules) pourraient intervenir comme le suggèrent les résultats sur la gangue interne isolée.

$L^{\prime}$ apparition fréquente de plusieurs arcs dans les réactions $L_{1}-_{1}$ comme dans les réactions $L_{2}-I_{2}$, est peut-être due à I'existence, dans chacun des deux cas, de plusieurs molécules différentes. Ces molécules auraient des propriétés très voisines puisque tous les arcs de type $L_{1}-I_{1}$ d'une part, ou tous ceux de type $L_{2}{ }^{-} I_{2}, L_{2}$ amidon, $L_{2}$-glycogène $d$ 'autre part, se comportent de la même manière dans les conditions expérimentales que nous avons utilisées. Ce résultat, ainsi que les communautés entre arcs de précipitation, nous ont d'ailleurs incités à donner le même symbole $\left(L_{2}\right)$ aux produits de l'oviducte antérieur qui réagissent avec $\mathrm{I}_{2}$ et avec l'amidon ou le glycogène. Mais on ne peut exclure l'existence de plus de deux lectines différentes dans I'oviducte antérieur du Pleurodèle.

Seul l'isolement des différentes molécules permettra de déterminer comment elles polymérisent entre elles et combien de lectines fonctionnelles différentes, sont excrétées. Le présent travail a d'ailleurs été réalisé dans cette perspective qui devrait nous permettre, à terme, de comprendre le rôle physiologique de ces molécules particulières. Par chromatographie d'affinité, nous avons déjà réussi à isoler une molécule appelée s.p.o.p. à l'époque (Jégo et Abalain, 1978). Le rendement de la purification de cette molécule qui est la lectine $L_{2}$, s'est avéré très mauvais à l'usage, ce qui nous a incité à réaliser l'étude des caractéristiques de ces réactions, rapportée dans le présent travail. II apparaît ainsi maintenant que la D-glucuronolactone que nous avions choisie pour réaliser les élutions, n'est pas un saccharide inhibiteur spécifique. Ce glucide inhibe pourtant très fortement la réaction globale OA-OM, sans que I'on sache exactement pourquoi (des résultats assez comparables ont été obtenus avec la concanavaline A qui ne reconnaît pourtant pas ce saccharide, elle non plus (Goldstein et Hayes, 1978). Les données nouvelles de ce travail devraient nous permettre d'améliorer très sensiblement l'efficacité de l'isolement de $L_{2}$; des expériences préliminaires réalisées sur de l'amidon insoluble avec une élution par la D-glucosamine, donnent des résultats très prometteurs.

Reçu en septembre 1982. Accepté en décembre 1982.

Remerciements. - Nous remercions très vivement Mesdames Allo et Bernard et Monsieur Blanchet, pour leur collaboration technique. 


\section{Références}

BATAILLON E., 1919. Analyse de l'activation par la technique des ceufs nus et la polyspermie expérimentale chez les Batraciens. Ann. Sci. nat. Zool., 10, 1-38.

BIRR C., HEDRICK J. L., 1979. Immunological identification of the jelly coat ligand for the Xenopus laevis cortical granule lectin. Fed. Prod., 38, 1252.

BOISSEAU C., 1973. Données autoradiographiques sur l'incorporation du sulfate de sodium radioactif dans l'oviducte de Pleurodeles waltiii Michah (Amphibien, Urodèle, Salamandridé). C. R. Soc. Biol. (Paris), 167, 746-750.

BOISSEAU C., JEGO P., JOLY J., PICHERAL B., 1974. Organisation et caractérisation histochimique des gangues ovulaires sécrétées par l'oviducte de Pleurodeles waltlii Michah. (Amphibien, Urodèle, Salamandridé). C. R. Soc. Biol. (Paris), 168, 1102-1107.

BOLOGNANI L., BOLOGNANI-FANTIN A. M., LUSIGNANI R., TONTA L., 1966. Presence of sialopolysaccharidic components in egg gelatinous mantle of Rana latastei and Bufo vulgaris. Experientia, 22, 601-603.

FOLKES B. F., GRANT R. A., JONES J. K. N., 1950. Frog-spawn mucin. J. chem. Soc., 440, 2136.

GOLDSTEIN I. J., HAYES C. E., 1978. The lectins : carbohydrate-binding proteins of plants and animals. In TIPSON R. S., HORTON D. Advances in carbohydrate chemistry and biochemistry, Vol. 35, 127-340. Acad. Press.

HUMPHRIES A. A., 1966. Observations on the deposition, structure and cytochemistry of the jelly envelopes of the egg of the newt, Triturus viridescens. Develop. Biol., 13, 214-230.

JÉGO P., 1974. Composition en glucides des différents segments de l'oviducte et des gangues ovulaires chez Pleurodeles waltiii Michah. (Amphibien, Urodèle). Comp. Bioch. Physiol., 47, 435-446.

JÉGO P., 1976. Analyse des protides des gangues ovulaires de Pleurodeles waltlii Michah. (Amphibien, Urodèle). Ann. Biol. anim. Bioch. Biophys., 16, 13-24.

JÉGO P., 1978. Analyse chimique et immunochimique des gangues ovulaires sécrétées par l'oviducte du Pleurodèle; effets de l'osstradiol sur les activités enzymatiques des cellules sécrétrices. Th. d'Etat, Univ. Rennes, sér. C, $\mathrm{n}^{\circ}$ série 80 .

JÉGO P., ABALAIN J. H., 1978. Isolement, par chromatographie d'affinité, d'une protéine spécifique apparaissant dans l'oviducte du Pleurodèle immature sous l'effet de l'œestradiol. Biol. cell., 31, 311-314.

JÉGO P., ABALAIN J. H., WROBLEWSKI H., 1976. Réactions de précipitation de type " antigèneanticorps ") entre produits de sécrétion de différentes régions de l'oviducte de Pleurodeles waltiii Michah. C. R. Acad. Sci. Paris, sér. D, 282, 767-770.

JÉGO P., CHESNEL A., JOLY J., 1983. Réactions de précipitation entre les produits de sécrétion des oviductes chez les Amphibiens. Reprod. Nutr. Dévelop., 23 (sous presse).

KATAGIRI C., 1973. Chemical analysis of toad eggs-jelly in relation to its "sperm capacitating " activity. Develop. Growth Different., 15. 81-92.

LEE P., 1967. Studies of frog oviducal jelly secretion. I. Chemical analysis of secretory product. Exp. Zool., 166, 99-106.

LOWRY O. H., ROSEBROUGH N. J., FARR A. L., RANDALL R. J., 1951. Protein measurement with the folin phenol reagent. J. biol. Chem., 193, 265-275.

MENDEZ B. A., LEFEBVRE M. J., OLAVARRIA J. M., 1968. La secrecion del oviducto del sapo Bufo arenarum : metodos para el aislamiento de precursores y estudio sobre su composicion. Acta physiol. latinoam., 18, 157-172.

METZ C. B., 1967. Gamete surface components and their role in fertilization. In METZ C. B., MONROY A., Fertilization : comparative morphology, biochemistry and immunology, vol. 1 , 163-236, Acad. Press, New York.

MINGANTI A., D'ANNA T., 1957. Ricerche sulla mucina ovulare di Triton cristatus. Ric. Sci., 27, 3052-3054.

MINGANTI A., D'ANNA T., 1958. Sulla composizione della mucina ovulare di Discoglossus pictus. Ric. Sci., 28, 2090-2093.

OUCHTERLONY O., 1949. Antigen-antibody reactions. Acta pathol. microbiol. scand., 26, 507-515. 
RIMINGTON C., 1940. Seromucoid and the bound carbohydrate of serum proteins. Biochem. J., 34, 931-940.

SOUPART P., NOYES R. W., 1964. Sialic acid as a component of the zona pellucida of the mammalian ovum. J. Reprod. Fert., 8, 251-253.

URIEL J., 1971. Color reactions for the identification of antigen-antibody precipitates in geis. In WILLIAMS C. A., CHASE M. W., Methods in immunology and immunochemistry, vol. III, 300-301, Acad. Press.

WOLF D. P., 1974. On the contents of the cortical granules from Xenopus laevis eggs. Dev. Biol., 38, 14-29.

WRIGHT C. S., 1980. Crystallographic elucidation of the saccharide binding mode in wheat germ agglutinin and its biological significance. J. mol. Biol., 141, 3, 267-291.

WYRICK R. E., NISHIHARA T., HEDRICK J. L., 1974. Agglutination of jelly coat granule components and the block to polyspermy in the Amphibian Xenopus laevis. Proc. nat. Acad. Sci, U.S.A., 71, 2067-2071.

YUREWICZ E. C., OLIPHANT G., HEDRICK J. L., 1975. The macromolecular composition of Xenophus laevis egg jelly coat. Biochemistry, 14, 3101-3107. 